\title{
Concertation et « figures de l'habitant » dans le discours des projets de renouvellement urbain
}

Dialogue and Representations of Residents in the Discourse of Urban Renewal Projects

Jacques Noyer et Bruno Raoul

\section{(QpenEdition Journals}

Édition électronique

URL : http://journals.openedition.org/edc/766

DOI : $10.4000 /$ edc.766

ISSN : 2101-0366

Éditeur

Université Lille-3

Édition imprimée

Date de publication : 1 décembre 2008

Pagination : 111-130

ISBN : 978-2-917562-00-0

ISSN : $1270-6841$

Référence électronique

Jacques Noyer et Bruno Raoul, «Concertation et « figures de l'habitant » dans le discours des projets de renouvellement urbain », Études de communication [En ligne], 31 | 2008, mis en ligne le 01 décembre 2010, consulté le 19 avril 2019. URL : http://journals.openedition.org/edc/766 ; DOI : 10.4000/edc.766

Ce document a été généré automatiquement le 19 avril 2019

(c) Tous droits réservés 


\section{Concertation et « figures de l'habitant " dans le discours des projets de renouvellement urbain}

Dialogue and Representations of Residents in the Discourse of Urban Renewal Projects

Jacques Noyer et Bruno Raoul

\section{Les cadres institutionnels incertains de la concertation}

1 En considérant les cadres qui régissent l'action publique dans le domaine du renouvellement urbain aujourd'hui, toute analyse visant à éclairer la problématique du rapport habitant/espace public est inévitablement amenée à s'intéresser à la place et l'emprise des dispositifs dits de concertation. Dans le règlement général de l'ANRU (Agence nationale de la rénovation urbaine) paru au journal officiel (3 avril 2007), la concertation est évoquée (plutôt que véritablement définie) de la façon suivante :

L'association des habitants et des usagers des quartiers concernés à l'élaboration du projet est indispensable pour répondre à leurs aspirations. Une concertation active repose sur :

- le partage du diagnostic et l'élaboration concertée du projet

- l'association des habitants et des usagers tout au long de la mise en œuvre du projet

- l'évaluation des effets de ce dernier auprès des habitants et des usagers.

2 Le caractère on ne peut plus général de ces indications - qui restent bien en deçà des préconisations formulées près d'un an plus tôt par le Comité d'Évaluation et de Suivi de l'ANRU qui faisait état d'un déficit de concertation entre les élus et la population concernée ${ }^{1}$ - laisse ouvert un champ d'action à la fois affirmé comme quelque chose d'essentiel et, en même temps, argumenté, si l'on peut dire, de façon particulièrement 
floue - dans un texte où, par ailleurs, le niveau de précision sur d'autres points relatifs aux questions d'habitat, d'aménagement et d'ingénierie des projets est, lui, particulièrement affiné. La "concertation» ne fait pas partie de ces mots nécessitant d'être explicités et les actes qui en traduisent la mise en œuvre sont symptomatiquement définis sous la reformulation tautologique de « l'élaboration concertée »... L'intention qui sous-tend ladite concertation, toute louable qu'elle puisse sembler: "répondre aux aspirations des habitants et des usagers des quartiers", ne donne pas plus lieu à explicitation, les aspirations en question semblant relever de l'évidence, ne nécessitant aucune forme d'argumentation. La référence à l'habitant donne l'impression que l'action publique serait ainsi naturellement légitimée.

3 Nous avons également pu relever cette imprécision au niveau local. En réponse à une préoccupation de l'IREV (Institut Régional de la Ville - Tourcoing) sur la question de la communication dans les projets de renouvellement urbain (PRU $)^{2}$ en région Nord/Pas-deCalais, nous avons en effet procédé ${ }^{3}$ courant 2007, à travers une enquête menée auprès d'une douzaine de sites de la région, à une première analyse exploratoire de documents de communication édités à ce sujet par des collectivités territoriales et à une exploitation des réponses obtenues à un questionnaire, administré par l'IREV, adressé à des responsables locaux de projets $\mathrm{RU}^{4}$. Cette première exploration du sujet nous permet de poser un cadrage en terme de problématisation qui, en l'état actuel de notre recherche, reste toutefois d'ordre programmatique.

Notre intention n'est pas, ici, de nous intéresser au fonctionnement du débat public dans les arènes de la concertation relative au renouvellement urbain. Notre objet est autre. Nous procédons à une analyse des formes du discours institutionnel pour saisir comment, dans l'espace public local, il met en scène et en sens la concertation. De ce fait, notre travail, en tant qu'il prend essentiellement pour matériau des productions écrites (des journaux, des magazines de collectivités...) qui constituent elles-mêmes un pan opératoire de démarches de communication, porte en définitive sur ce que l'on pourrait considérer comme " communication de la concertation", dont le discours est par conséquent inclus lui-même dans une stratégie où il a sa place propre et une efficacité recherchée. Au demeurant, les deux notions de concertation et de communication sont fortement intriquées tant dans les textes officiels que dans les différents discours et documents émanant des sites mobilisés sur ces projets.

Dans le cadre de cet article, nous mettrons l'accent sur deux axes structurants de la problématique telle que nous la voyons émerger à partir de notre exploration. Il en ressort notamment que, notion courante, sinon récurrente dans les documents des collectivités locales, la « concertation » apparaît en définitive comme un point de passage obligé du discours sur le renouvellement urbain sans que son objet, dans l'ensemble, ne soit rendu très explicite, sans qu'on n'en sache ni la portée ni l'intention qui la fonde. Cela dit, l'intérêt d'une démarche inductive, depuis les documents de terrain, est de faire remonter des sites - de leurs logiques d'inventivité comme de leurs attitudes de frilosité ce que sont les registres discursifs et les gammes de mise en pratique d'une concertation laissée à l'initiative in situ des concepteurs de projets. C'est donc, autant que l'usage de la notion de concertation, les formes et enjeux du métadiscours sur cette notion qu'il s'agira ici de prendre en compte et d'analyser, conjointement aux représentations auxquelles renvoient ces formes, aux systèmes de places des acteurs vers lesquels elles orientent ainsi qu'aux « compétences » respectives qu'elles reconnaissent ou attribuent. 
Plus particulièrement, nous nous interrogerons sur la place réservée ou faite aux habitants - c'est là le deuxième point central de notre réflexion -, sachant que, derrière la notion de concertation, cette place est bien souvent affirmée comme étant au centre du dispositif. Si l'analyste peut s'interroger sur les représentations du public qui sont au fondement de tels discours, le champ de questionnement ici ouvert concerne la manière dont l'individu-destinataire de ces opérations est construit, c'est-à-dire "figuré » et « projeté » dans les actes et par les discours qui le prennent en compte, «segmenté » et organisé en figures autonomes (ou non) selon les phases de l'opération - quand bien même, et paradoxalement d'une certaine manière, la parole des habitants se révèle peu convoquée dans les documents.

7 A partir, par conséquent, d'une approche de la concertation comme notion fondatrice des projets de renouvellement urbain et paradoxalement empreinte d'une absence de cadrage spécifique (notamment opérationnel), il nous faudra observer comment, en considérant les représentations construites de l'habitant, les différentes échelles du collectif«nucléaire » (la famille), localisé (le quartier), global (la ville) - interviennent dans ces projets, orientant vers des conceptions parfois fort différentes de ce qu'est l'acte de concertation - dont on se souviendra qu'étymologiquement, il est apparenté à la notion de lutte.

\section{La concertation comme passage obligé dans le discours des PRU : une lecture communicationnelle d'une dimension de l'espace public urbain}

\section{«Formes » de ce discours et représentations sous-jacentes : une concertation de marge}

8 L'association des habitants à l'élaboration d'un projet de renouvellement urbain dans son ensemble est donc officiellement présentée, aujourd'hui, comme étant indispensable. Implicitement, paraît ainsi reconnue la part de «sujet pensant » de l'habitant dans une étape amont d'un processus d'élaboration de l'action publique locale : selon les textes de cadrage, l'habitant n'est pas envisagé sous le seul statut d'usager (d'un espace collectif, d'un service...) ou d'occupant (d'un logement), sous le seul statut de cible ou de bénéficiaire de l'action menée, du projet en cours. En rupture avec le mode de penser qui a prévalu au moment de la construction des grands ensembles (Peillon, 2001) ${ }^{5}$, cette forme de reconnaissance du point de vue des habitants (entendus ici comme sujet collectif) tient d'un nouveau répertoire de l'action publique (Gaudin, 1999), elle se comprend à l'aune de l'institutionnalisation de la participation au cours des quinze dernières années (Blondiaux, 2004 ; Lefebvre, Nonjon, 2003), elle vient s'inscrire dans le contexte de l'affirmation de «l'impératif délibératif » (Blondiaux et Sintomer, 2002). Ceci dit, pour de nombreux militants de la cause participative, la concertation n'est pas à confondre avec la participation, elle n'en est qu'une étape, certes indispensable, pour y arriver : par la concertation, explique ainsi Georges Gontcharoff, militant de longue date de la démocratie participative et conseiller de la revue Territoires, "les citoyens sont appelés à être associés à l'élaboration d'un dossier, au traitement d'une question, avant la décision qui continue de relever du pouvoir élu. Par elle, le pouvoir s'enrichit des points de vue, des 'compétences' des 'forces vives' qu'il associe à l'instruction de sa décision. 
Mais les instances de concertation ne sont pas des instances de décision » (Gontcharoff, 1999, 313). D'autre part, en mettant en avant cette question de la concertation, les textes institutionnels entendent faire valoir la démarche de projet et non pas uniquement l'objet du projet, sa finalité concrète, à savoir la transformation physique du quartier. Le projet s'entend alors comme "activité sociale continue», la concertation participant d'un processus par lequel l'action publique urbaine travaille à la régulation consensuelle (Pinson, 2005).

Mais, des documents de terrain, c'est une réalité autre qui se dégage de la manière dont est mise en discours et en sens la concertation. Ainsi, dans un encadré en fin d'une brochure municipale, une instance spécifiquement créée dans le cadre du projet de renouvellement urbain est-elle présentée, à l'adresse des habitants du quartier concerné, comme «instance de concertation et de présentation des projets »: «des rendez-vous réguliers vous permettent de venir poser vos questions et vous renseigner sur les aménagements en cours et à venir ». Les habitants sont donc informés qu'ils peuvent venir... s'informer, se renseigner. Ils sont donc mis dans une posture précise, celle de personnes souhaitant être informées et poser des questions sur un projet qui les concerne au premier chef, mais ils ne sont pas explicitement placés dans la posture d'interlocuteurs. Cette remarque, toutefois, pourrait être nuancée en se référant à ce qui suit dans cet encadré : le Point Info mensuel, est-il dit, est un « lieu ouvert à tous permet [tant] aux élus, techniciens et habitants de se rencontrer aussi pour discuter en toute simplicité et convivialité, des problèmes du quartier ». Deux observations concernant une telle instance de concertation: outre que les problèmes du quartier sont présentés comme objet de simple discussion et non comme objet de délibération c'est-à-dire comme points susceptibles de conflictualité, on pourra relever que, en l'occurrence, ce n'est pas le projet en tant que tel qui est dit être mis en débat; d'autre part, tout le monde étant, aux termes de l'énoncé, situé sur un pied d'égalité, l'implicite du propos de la communication institutionnelle ici est que les élus et techniciens feraient leur "l'égalisation de la condition des participants» (Lefebvre, 2007), quand bien même chacun est assigné à son rôle social- les compétences et les responsabilités étant implicitement attribuées et délimitées par le texte - et alors même que ce texte institutionnel n'envisage pas la figure du «citoyen en tant qu'habitant»- en paraphrasant une formule de J. M. Fourniau (2007). En d'autres termes, les habitants ne sont pas élevés en sujet politique.

Dans une autre ville, à travers la mise en place d'«ateliers » qui sont explicitement présentés comme "nouvelle instance de démocratie participative ouverte aux habitants ", est évoqué le principe "d'associer les habitants au suivi quotidien de cette rénovation urbaine ». Ici non plus, l'arène publique n'est pas à confondre avec un forum d'échanges ouvert à tout un chacun à propos des fondements et des orientations du projet. En somme, le débat public ici ne semble pas permettre l'ouverture d'un moment d'incertitude dans la dynamique de la conduite de projet (Simard et Fourniau, 2007). Dans un autre endroit encore, sous l'intertitre " une participation citoyenne », il est bien dit qu'

un tel travail ne peut se faire sans une concertation active et régulière avec les habitants [mise en place d'ateliers urbains] [...]. Dans un souci de pédagogie interactive, les citoyens sont invités à faire part de leurs besoins et de leurs attentes sur des points très précis, qui font partie de leur quotidien. Certains quartiers sont très demandeurs, comme en témoignent les nombreux appels reçus au Numéro 
Vert mis en place pour rassembler les requêtes des habitants. La concertation est de

plus en plus forte.

11 peut avoir l'impression ici d'un glissement: l'idée affichée de participation et concertation interactive s'entend, sur un plan pratique, en termes de prise en compte des requêtes, mais pas en termes de réelle contribution des habitants à l'élaboration d'un "projet», on n'est pas dans l'idée qu'ils "puissent peser sur l'orientation des constructions et des réhabilitations", un objectif qui constituait, nous dit Jacques Donzelot, la raison d'être des politiques de Développement Social Urbain menées à partir des années 1980 en France (Donzelot, 2003, 222). parce qu'intervenant en aval, sur un projet déjà longuement élaboré, est couramment circonscrite à des domaines délimités et limités, les places occupées dans ces moments publics ne semblant guère permettre aux habitants-citoyens d'investir des rôles véritablement propositionnels, sur des points centraux - ce qui semble les amener à fréquemment intégrer cette place marginale à laquelle la situation construite les invite et à faire, en quelque sorte, des suggestions "à la marge ", sur de " petits objets » : cadre de vie, aires de jeux, espaces verts, aménagement de place, résidentialisation... En d'autres termes, la communication institutionnelle locale laisse deviner une grande prudence en matière de concertation qui, paradoxalement, à l'aune des représentations du « local » comme «petite madeleine de la démocratie » (Wolton, 2000), donne force d'ambition démocratique au discours de l'ANRU.

\section{L'emprise du faire savoir : la concertation comme procédure de « mise en acceptation»}

13 Toute contributive qu'elle ait été, dans leur ensemble, les textes des documents de communication ne permettent pas de savoir en quoi il y a eu contribution des habitants. On peut ici émettre l'hypothèse d'une difficulté de la part des services communication des institutions à en rendre compte, voire à prendre en compte une telle contribution, à moins qu'il ne s'agisse d'un impensé, ou le signe que, pour ces services, il n'y a pas grand chose à en retirer et donc à en dire. Par exemple, dans un article (une page entière d'un magazine municipal) relatif à une réunion publique à vocation informative, il est fait état d'une concertation à venir à propos d'une place à aménager. A la fin de l'article est évoquée la prise de parole des habitants, mais sans que n'en soit indiqué l'objet : « Après la présentation de ces différents projets, ont suivi les questions des habitants auxquelles le maire et les élus présents ont répondu lors d'un débat constructif et chaleureux ». En somme, la conclusion fait dans la formule rassurante... mais évasive, le lecteur ne saura rien de la teneur des questions et des axes du débat, hormis l'évaluation positive et consensuelle du cadre dans lequel ils se sont déroulés. Dans un autre endroit encore, sous l'intertitre "Écoute et concertation », on peut lire: «Pendant toute la phase d'études préalables, de nombreuses rencontres ont eu lieu, un diagnostic précis a été établi. Encore récemment, une réunion publique a eu lieu permettant à tous les habitants de donner leur avis et d'obtenir des réponses à leurs questions ». Ici non plus, on ne verra pas en quoi il y a eu échange public, en quoi et sur quoi il y a eu concertation.

14 En définitive, globalement, derrière la notion, prédomine ce que l'on pourrait appeler le « registre informatif ». Dans certains endroits, le mot donne l'impression de faire alors

Études de communication, 31 | 2008 
office, en lui-même, de caution de démocratisation de la procédure (performatif) et de légitimation de l'action. Mais, plus concrètement, il ne semble pas y avoir une autre ambition à ce sujet. Au fond, le registre de l'information sur lequel est fréquemment déclinée la démarche laisse apparaître une conception implicite de la concertation comme « descendante », fortement fonctionnelle... Au demeurant, le directeur général de l'ANRU, Philippe Van de Maele, ne laisse pas entendre un autre raisonnement dans la réponse qu'il formule à la Gazette des communes (mars 2005) :

-GDC: La communication et la concertation avec les habitants sont-elles suffisantes?

- PVDM : Pas toujours. Or les programmes de rénovation urbaine revêtent une dimension sociale et psychologique très importante. La concertation relève quasiment d'une obligation morale et elle est la clé du succès d'une opération. Le rôle des bailleurs sociaux est important de ce point de vue. Informer, expliquer, rassurer les habitants sur leur parcours résidentiel, sur la transformation de leur quartier, sur le calendrier de ces transformations sont loin d'être superflus. C'est capital.

On voit de manière très explicite comment est entendu ici l'objet de la concertation: celle-ci relève d'une démarche consistant à faire savoir ce qui a déjà été décidé et d'une logique de négociation opérationnelle d'un projet. Cette idée d'expliquer mais plus encore de rassurer est tout à fait symptomatique d'une certaine représentation du rapport concepteurs/habitants qui prévaut dans les instances nationales ainsi qu'au niveau local ${ }^{6}$, la «dimension psychologique» dont il est fait état faisant résonner une figure particulière de l'habitant, celle d'une personne inquiète à laquelle les porteurs de projet et les opérateurs se doivent d'être attentifs.

$\mathrm{Au}$ terme de cette première étape de notre réflexion, on peut se demander dans quelle mesure les discours et actions autour de la concertation dans les PRU ne relèvent pas d'une certaine forme de "disciplinarisation" de la fonction habitante. A l'heure de l'« impératif délibératif ", il y aurait une sorte d'injonction à la participation mettant l'habitant dans une position de devoir donner son avis à propos d'un projet de transformation de son propre espace de vie quotidien mais sur lequel il n'a, fondamentalement, aucune emprise. In fine, on pourrait dire, à travers la récurrence d'un tel terme, que l'on se trouve fréquemment dans une logique de « mise en acceptation » du projet plutôt que dans une logique de mise en débat de ce projet. «La participation, nous dit de son côté Rémi Lefebvre, est surtout conçue comme une aide à la décision et à la construction de l'acceptabilité sociale des projets » (Lefebvre, 2007, 207). En aucun cas, la concertation ne semble porter sur le projet de renouvellement urbain comme « projet de territoire ». Par exemple, dans les documents recueillis, nous n'avons pas repéré de lieu où auraient été soumis à discussion publique la pertinence ou pas et le souhait ou pas des habitants de procéder à telle ou telle démolition. Du reste, du côté de certains concepteurs, une telle délimitation peut être pleinement assumée. L'extrait d'entretien avec l'architecte Roland Castro publié dans la Lettre des Forums régionaux des acteurs de la rénovation urbaine (septembre 2006) est en ce sens tout à fait explicite :

Question : En tant que concepteur, quel parti avez-vous tiré des solidarités existant entre les habitants?

- RC: Nous ne sommes pas particulièrement en contact avec les gens dans le processus de formalisation du projet. C'est davantage le rôle de l'équipe de projet. Par contre, la démarche de transformation du quartier est bien accompagnée et animée. La conception du projet urbain global est un geste assez volontaire dans lequel un architecte un peu metteur en scène pose des actes. Cela ne passe pas par 
une forme de démocratie participative très développée. On le présente, on le

défend, c'est tout ${ }^{7}$.

habitants des lieux, elle définit aussi la problématique de l'articulation de la professionnalité et de la technicité avec le savoir profane (ou non-professionnel serait-il plus exact de dire), elle pose la question de la possibilité et de la pertinence pour les concepteurs de composer avec « l'expertise d'usage » de l'habitant. Raisonner de la sorte permet d'envisager la question de la concertation dans des termes autres que ceux de pouvoir/contre-pouvoir. La concertation ainsi entendue est par conséquent une forme de réassurance non pas tant des habitants (inquiets du devenir de leur lieu) que des concepteurs (inquiets du devenir de leur projet). Cela dit, considérant que concerter n'est pas décider, c'est aussi la question de la responsabilité politique qui affleure ici.

\section{La figure de l'habitant dans les PRU : sur quelques jeux identitaires}

\section{L'habitant projeté dans un quartier reconfiguré}

18 Si le projet, ainsi que l'évoquent M. Barthélémy et L. Quéré $(1995,292)$, est cette « action possible et désirable »- et, ici, précisons-le, rendue possible dans le cadre d'une politique publique - il est sans doute important de souligner que ce possible a à être construit comme «désirable » et que c'est là, probablement, que la communication (de projet) a un rôle important à jouer ainsi que les différentes formes de concertation mobilisables.

Cette communication accompagnant la genèse et l'évolution du projet ne peut faire l'économie, y compris dans les phases de conception les plus initiales, de celui qui est présenté comme étant appelé à être un des acteurs principaux du processus : l'habitant. Ainsi peut-on lire par exemple, sous l'intertitre «Les habitants au cœur du projet », dans le chapeau d'un dossier présenté en une d'un magazine municipal: "Ce grand projet 'pour' les [habitants de la ville] sera réalisé 'avec' eux ». L'éditorial du maire pourra faire dans l'interpellation directe: "Rien ne se fera sans vous. Ce projet est à réaliser ensemble. Vous savez combien je suis attachée à vos avis, vos remarques, vos suggestions ». En convoquant en ces termes la figure de l'habitant interlocuteur, le discours institutionnel fait ici valoir l'idée que le chantier du renouvellement urbain passe par une relation personnalisée entre l'édile et ses administrés.

En même temps qu'un espace urbain est inscrit dans une dynamique de projet, il apparaît que l'habitant des lieux est, lui aussi, projeté: imaginé comme résident potentiel, représenté comme usager de ces lieux nouveaux qu'il lui faudra investir. Le rapport à l'imaginaire est ici, sans doute, un élément tout à fait essentiel : « Pour notre quartier, le renouvellement urbain va renforcer le bonheur de vivre ensemble" (conseillère municipale) ; le quartier « va devenir une zone résidentielle recherchée »; « les habitants du quartier savent désormais qu'ils participent à une des plus belles aventures et à un des plus beaux projets que la commune a connus »; « l'ensemble du quartier [...] va connaître une seconde naissance "; "Un projet qui a séduit l'Agence nationale de rénovation urbaine qui a vu là une véritable démarche pour réinventer la ville et non pas une simple opération de démolition-reconstruction ». L'imaginaire du quartier nouveau (appuyé fréquemment sur la mise à l'écart, dans les mentalités, des déficits et stigmates divers liés à l'ancien $)^{8}$ nécessite des modes d'identification à un espace urbain non encore existant. 
Le lecteur pourra ainsi se laisser enthousiasmer par le discours institutionnel local... ou se montrer très sceptique devant une conception pétrifiante de l'urbanité, à l'heure d'une forte incitation de toutes parts à la mobilité: «Le quartier [...] vit une véritable transfiguration. On sera fiers d'y habiter. Assis sur un muret, quelques jeunes 'tapent la discut' et projettent leur avenir dans ces immeubles où ils créeront certainement une famille ». Le renouvellement urbain est figuré au travers de ces projections individuelles et de ces micro-récits où la fondation d'une famille et l'avenir des enfants sont souvent des ingrédients majeurs : l'abstraction technique du dossier trouve sans doute là un mode de traduction - ou de compensation - directement relié au vécu et aux projets individuels des habitants. Si la figuration, par des maquettes ou des plans paysagers, du quartier nouveau accomplit une forme de réalisation matérielle de cet imaginaire, il est manifeste que l'habitant, aux différentes phases de conception et de mise en œuvre du projet, est cadré comme partenaire du renouveau, pour reprendre la formule d'un maire se félicitant "de l'adhésion des habitants » : " 'avec vous, nous allons changer l'image du quartier' ».

21 L'argument - implicite ou explicite - de la démocratie participative où parler, être écouté, donner son avis... participent du lien d'une politique en phase, sur le terrain, avec le quotidien des gens, leurs préoccupations les plus immédiates, est quelque chose qui parcourt nombre de documents de communication sur les sites étudiés. Lié aux questions d'image du quartier et de "qualité », ce modèle d'un habitant en adéquation avec les bienfaits du projet vient, dans un certain nombre de publications (et de documents de communication) s'imposer comme la figure-type de l'habitant du quartier, non pas oppositionnel-comme la concertation pourrait le laisser supposer- mais en convergence fréquente avec les projets développés. Peut-être peut-on, à ce propos, parler d'habitant-argument: celui au nom duquel et au bénéfice duquel le pouvoir local justifie d'un projet ou de décisions - souvent préfigurées - relatives à ce projet. L'enjeu est alors la coïncidence entre l'habitant projeté et celui, bien réel celui-là, que les concepteurs du projet sont amenés à rencontrer - notamment lors de ces moments de concertation. Et ce sont les formes de décalage entre le bénéfice imaginé pour le futur habitant et les éventuels nuisances/inconvénients identifiés par ceux qui seront concernés qui renforcent parfois l'incompréhension là où elle était censée se réduire.

Dans l'argumentaire institutionnel mobilisé par les décideurs locaux, la question de la réintégration $\mathrm{du}$ quartier soumis à renouvellement dans le tissu urbain général est fréquemment un objectif central : il s'agit de «favoriser l'intégration du quartier dans la ville», de lui «faire retrouver une nouvelle centralité ». A la logique subie de la relégation, on essaie de substituer une dynamique d'intégration du quartier dans la ville signifiant la volonté de rompre avec les modèles antérieurs de cloisonnement entre les populations. Est-ce à dire, pour autant, qu'une telle volonté est susceptible, à elle seule, de réduire les images négatives du quartier - en premier lieu dans les représentations mêmes de ceux qui y vivent? On ne rompt pas aussi facilement, croyons-nous, avec ces représentations stigmatisantes, sédimentées de longue date, dans et hors les murs du quartier, et force est de constater que les stigmates sociaux continuent à se faire entendre de multiples façons dans ces moments de concertation'. L'habitant de ces quartiers est donc d'abord vu à partir de l'image externe du quartier: selon une sorte de pré-identité collective qui, en quelque sorte, prédomine sur l'identité individuelle - et parfois même sur l'image de soi - au point de ne laisser entendre, dans l'« élaboration concertée du projet » (ANRU), que le mal-être d'une identité collective subie dans laquelle l'habitant des lieux ne se reconnaît pas. 


\section{De l'adversatif...} qu'elle conditionne, de multiples façons, les démarches de concertation entreprises : nous voulons parler ici de ce que nous appellerons l'habitant protestataire. Les protestations et les contestations qui trouvent (ou ne trouvent pas) à s'exprimer ont certes des origines diverses, liées de façon générique à des nuisances subies que le projet - dans sa phase de démolition, notamment - induit ${ }^{10}$. Les réunions ou ateliers de concertation ont alors tendance à devenir des lieux où il est avant tout question - paradoxalement mais aussi symboliquement - de regretter qu'elles n'aient pas eu lieu avant et de « vider son sac ». La focalisation s'exerce alors sur les nuisances personnellement subies: l'échelle de perception du projet réduit ce qui est de l'ordre de l'intérêt général (avec inconvénients ponctuels) à une identification de la situation en termes d'intérêt particulier, dégradé par un certain nombre de nuisances, mal vécues par effet de cumul.

La logique globale du projet urbain est recadrée en perception située, localisée où, de générique en spécifique, ce sont avant tout les inconvénients immédiats de ces projets (perçus comme) lointains qui sont mis en avant par un locataire se ressentant sous l'emprise de décisions qui, tout en le concernant, le dépassent et sur lesquels il ne se sent avoir qu'une influence marginale. Nous croyons que le sentiment fréquent d'exclusion du citoyen-habitant de la phase définitionnelle du projet est un élément explicatif du prisme réduit de perception de la portée de ces projets par ceux qu'ils concernent. L'argument de l'intérêt général mobilisé comme argument justificatif par les concepteurs du projet pour la ville (ce que l'on pourrait appeler "l'intérêt général global») et pour le quartier ( l'intérêt général spécifique »), faute d'être construit dans le croisement des discours et des perceptions en phase de conception, fait retour sur le mode systématique de l'insatisfaction en aval de ce qui a été déjà conçu.

Dès lors, ne s'entend guère que cette position adversative, qui, fréquemment intégrée par les porteurs de projets, engendre ce que l'on pourrait appeler une «conception préventive » de ces moments de concertation. Préventive dans le sens où la concertation vise alors essentiellement à estomper par avance ce qui est pressenti comme conflictuel : une tension sur tel ou tel point étant prévisible, la concertation a pour objet de réduire cette tension, de faire baisser la pression. Produire de l'apaisement - à moins qu'il ne s'agisse de l'installation d'une forme de silence ainsi interprétable - comme objectif récurrent de ces moments de rencontre conduit donc à dé-placer l'acte de concertation : de l'écoute programmée des habitants pour recenser les difficultés et les points d'achoppement, on aurait tendance à passer à l'anticipation des points de conflit potentiels pour les réduire dans l'acte de communication qu'est la concertation.

Il est probablement essentiel de traiter ces questions en termes de décalages de temporalités : là où élus et techniciens sont orientés vers un futur du territoire et vers des dimensions à la fois prospectives et projectives - tributaires de calendriers divers et de contraintes liées à des échéanciers tant techniques que politiques - l'habitant est plutôt centré sur le vécu quotidien, et les résurgences diverses d'un passé « résidentiel » plus ou moins bien vécu. On peut d'ailleurs parfois se demander s'il n'y a pas l'idée d'une mise à l'écart de l'habitant comme condition de l'avancée technique de ces dossiers, ce qui expliquerait, au moins partiellement, ce ressenti régulièrement évoqué du côté des destinataires de ces projets d'une concertation « trop tardive ». 

par l'ANRU pour satisfaire à la conformité de l'avancée des projets et du potentiel de fragilisation de ces projets que représente leur soumission à la discussion et aux forts infléchissements qui risquent d'en résulter. Que les habitants, dans ces conditions, perçoivent les élus et les techniciens comme des alliés objectifs, peu spontanément disponibles à une fragilisation possible - voire probable - du projet par la concertation ne saurait donc totalement étonner...

Un autre élément joue probablement dans ce processus : le cumul des cultures techniques et des langages experts, dans de tels sujets, est souvent un élément de mise à distance ou d'inaudibilité de la parole " profane »/non-professionnelle de celui qui sera pourtant au centre du projet devenu réalité. Il y a là une sorte de paradoxe fondateur des intentions de l'ANRU, selon nous, en matière d'attention accrue aux savoirs dont dispose l'usager des lieux voués à renouvellement : cette parole venue du cœur de l'usage est souhaitée sans qu'on éclaircisse précisément les conditions de son audibilité nouvelle.

\section{... Au contributif}

Il est pourtant non négligeable de prendre en compte dans ces questions reliées aux démarches de concertation ce qu'il est convenu d'appeler l'expertise d'usage. «Partenaire du renouveau » local, l'habitant est aussi un usager-expert des lieux. Cette forme d'expertise contribue à créer sa compétence particulière de partenaire, de conseil... En tant qu'usager quotidien d'un espace, il est le praticien de la réalité quotidienne de ce lieu, capable de faire valoir un point de vue ancré dans les préoccupations ordinaires du vécu. En ce sens, l'habitant/usager est un expert : expert en tant que détecteur de difficultés particulières, utilisateur averti d'un espace, élément de veille et diagnostic des besoins spécifiques d'un quartier, d'un lieu... Mais, on le sait, cette forme d'expertise peut n'être que faiblement audible en comparaison d'expertises plus techniques et apparaissant plus en amont des projets de renouveau. Cette forme d'expertise trouve, aux yeux de bon nombre de décideurs, ses limites devant la technicité et la professionnalité que requiert un PRU.

C'est, dès lors, à un autre niveau de " concertation » - qui n'a plus rien de public - que l' habitant en instance (celui qui est en situation potentielle de relogement) devient partenaire d'une négociation pour l'accès au futur logement. Cet habitant en devenir peut être considéré, à ce moment particulier de son parcours vers le "logement rénové", comme un «contractant social " (Zémor). On se situe ici, plutôt du côté des logiques du bailleur: "chartes de relogement", "chartes de bonne occupation", "Parcours Résidentiel Adapté » (PRA) apparaissent comme autant de contrats, plus ou moins formalisés, mais qui fixent les cadres d'engagements réciproques, en fonction d'un intérêt (voulu comme) général. Ces éléments contractuels, même lorsqu'ils ne s'appellent pas " chartes » apparaissent comme une sorte d'arrière-plan constant de la concertation où l'on vise à contribuer à stabiliser des engagements de part et d'autre sur ce qui, par définition, est instable puisqu'en discussion... On peut alors, sans doute, parler de "concertation-micro»: le rôle du chargé de mission relogement y est crucial et la logique d'accompagnement individualisé qui prévaut alors est généralement envisagée sous la forme d'un travail sur le « projet de vie » et non simplement sur le relogement. On entre, par un choix spécifique de posture du bailleur, dans l'intimité des familles - et dans l'espace privé comme composante de l'urbain - où le passage de l'espace habité à l'espace à

Études de communication, 31 | 2008 
habiter s'opère, dans ce moment transitionnel, par ces modes d'ajustement où les intérêts du bailleur et ceux du locataire tentent de se rejoindre.

On voit bien que nous ne sommes jamais loin, avec ce type de question, de l'identité individuelle et collective, que cette problématique du renouvellement urbain effleure constamment. Cet habitant transitoire, pris entre démolition et relogement, est, si l'on reprend la logique dénominative des bailleurs, un "résident » : à savoir une personne qui occupe depuis un certain temps un logement, dans un lieu donné, sujet à « renouveau ». Cette personne est donc définissable, d'un premier point de vue, par son "passé résidentiel ", son histoire propre avec ce lieu et l'histoire de ce lieu (dont elle est, au fond, un fragment) ${ }^{11}$. La question se pose alors de savoir dans quel contexte, partie de l'histoire de la ville, s'inscrit le quartier et, de fait, le projet qui y "prend place». On peut constater, sur les sites travaillés, que cette dimension n'est pas ignorée et que les ateliersmémoire de quartier sont sans doute un point sensible - et non-paradoxal - du processus communicationnel lorsqu'il s'agit de renouvellement urbain. Il faut sans doute souligner, à cet égard, l'importance de ce que l'on pourrait appeler le «travail de deuil » du résident, que la communication sur ces projets a à construire. En même temps que le relogement, il y a ce que l'on pourrait appeler le " dé-logement » (lié, naturellement, à la problématique de la démolition d'immeubles existants, d'habitats indignes...). Subi ou accepté, le fait d'être dé-logé et la perception qui va être construite autour de ce basculement d'un passé à un futur semblent fondateurs de ces projets de rénovation : l'acceptabilité du nouveau n'est sans doute pas qu'affaire d'aménagement et de technicité, elle est aussi conditionnée par un travail relationnel, communicationnel sur le "deuil de l'ancien ", c'est-à-dire sur l'histoire dont il est empreint, sur le patrimoine individuel et collectif qu'il constituait, sur les trajets de vie dont il est chargé.

Sous la face apparente de l'habitant, ce sont bien les figures multiples de cette identité individuelle et collective, ancrée dans l'histoire d'un lieu et ayant à se projeter dans les reconfigurations de cet espace, que la concertation des projets de RU a à prendre en compte. Si la distinction de ces figures - non exclusives l'une de l'autre et dont la coexistence dans les situations diverses de concertation fait partie de stratégies de mise en audibilité des caractéristiques multiples de la place habitante - est essentielle pour identifier les enjeux des différentes formes de concertation mises en œuvre, il n'en reste pas moins, après le parcours analytique entrepris sur les sites étudiés, que la notion de concertation, prise à la fois dans des temporalités complexes, dans des stratégies de restructuration de territoires et leurs implications symboliques, dans les enjeux politiques locaux et leurs ramifications nationales... ouvre, de par son flou définitionnel, sur un jeu duquel les acteurs qui la promeuvent tirent parti pour signifier volonté d'écoute, d'attention, de prise en compte et faire valoir l'image d'une institutionnalisation consensuelle de la concertation. En même temps, nous l'avons vu, il apparait que communiquer sur l'acte de "concertation" est davantage de l'ordre performatif de l'affirmation d'un lien, d'une écoute régulièrement entretenue optimisant l'acceptabilité du projet plutôt que de l'ordre du débat contradictoire susceptible de le freiner ou d'en handicaper les phases programmées d'accomplissement. En d'autres termes, la " communication de la concertation » révèle une démarche enchâssée dans une perspective fonctionnaliste, le moment public du débat s'y entendant comme moment d'entérinement et non comme moment communicationnel de production d'un neuf. L'objet de la concertation se décline alors en forme d'accréditation de la communication de projet bien plus qu'en prise de risque démocratique. 
a dit, la communication institutionnelle ne saurait contenir la dynamique communicationnelle du débat public sur les changements dans (de) la ville. En explorant, parallèlement à notre analyse des documents des collectivités territoriales, du côté de la presse quotidienne régionale ou de journaux associatifs de quartier qui ont leur manière de faire savoir et faire valoir le point de vue d'habitants, nous avons pu le constater, la thématique du renouvellement urbain peut échapper au consensus, elle est matière à conflit. Ressort là une autre piste d'investigation - que l'on suggère en guise d'ouverture conclusive pour notre papier et, dans le même mouvement, en prolongement de notre travail en cours - : il s'agira de voir en quoi la question du renouvellement urbain et celle, corrélée, de la concertation donnent lieu, via la matière discursive de productions écrites de différents statuts, à interaction sociale et, plus spécifiquement, en quoi elles font (peuvent faire) l'objet de controverse dans l'espace public local.

\section{BIBLIOGRAPHIE}

Barthélémy, M. et Quéré, L., (1995), «Les enquêtes d'utilité publique : une production collective? », in : Quin C. (dir.), L'Administration de l'équipement et ses usagers, Paris, La Documentation Française, pp. 281-299.

Blondiaux, L., (2004), « Démocratie délibérative et démocratie participative : une lecture critique » (à paraître dans Bacqué M. H. et Sintomer Y. (éds.), Démocratie participative et gestion de proximité, Paris, La Découverte), Conférences de la Chaire MCD (Mondialisation, Citoyenneté et Démocratie), conférence prononcée à l'Université du Québec à Montréal, 11 novembre 2004.

Blondiaux, L. et Sintomer, Y., (2002), «L'impératif délibératif », Politix, vol. 15, n 57, pp. 17-35. Donzelot, J., (2003), Faire société. La politique de la ville aux États-Unis et en France, Paris, Seuil, 366 p. Fourniau, J. M., (2007), «'Citoyen en tant que riverain' : une subjectivation politique dans le processus de mise en discussion publique des projets d'aménagement », in : Revel M., Blatrix C., Blondiaux L. et alii (dir.), Le débat public : une expérience française de démocratie participative, Paris, La Découverte, pp. 67-77.

Gaudin, J. P., (1999), « L'espace public des politiques contractuelles », pp. 231-247, in : François B. et Neveu É. (dir.), Espaces publics mosaïques, Rennes, Presses Universitaires de Rennes, 322 p., pp. 231-247.

Gontcharoff, G., (1999), « Le renouveau des comités de quartier », in : CURAPP/CRAPS, La démocratie locale. Représentation, participation et espace public, Paris, P.U.F., 424 p., pp. 305-327.

Lefebvre, R. et Nonjon, M., (2003), « La démocratie locale en France. Ressorts et usages », Sciences de la Société, $\mathrm{n}^{\circ}$ 60, pp. 9-28.

Lefebvre, R., (2007), « Les élus : des acteurs peu dialogiques du débat public », in : Revel M., Blatrix C., Blondiaux L. et alii (dir.), Le débat public : une expérience française de démocratie participative, Paris, La Découverte, pp. 207-218.

Peillon, P., (2001), Utopie et désordres urbains. Essai sur les grands ensembles d'habitation, Paris, Éditions de l'aube, $286 \mathrm{p}$. 
Pinson, G., (2005), « L'idéologie des projets urbains. L'analyse des politiques urbaines entre précédent anglo-saxon et 'détour' italien », Sciences de la Société, nº 65, pp. 29-51.

Quéré, L., (1995), « L'espace public comme forme et comme événement », in : Joseph I. (dir.), Prendre place, Plan Urbain/Édition Recherches, pp. 93-110.

Quéré, L., (2000), «Au juste, qu'est-ce que l'information? », Réseaux, n 100, CNET/Hermes Science Publications, pp. 331-357.

Simard, L. et Fourniau, J. M., (2007), « Ce que débattre nous apprend. Éléments pour une évaluation des apprentissages liés au débat public », in : Revel M., Blatrix C., Blondiaux L. et alii (dir.), Le débat public : une expérience française de démocratie participative, Paris, $\mathrm{La}$ Découverte, pp. 318-331.

Tripier, P., Jacques-Jouvenot, D., Spadone, P.-L. et Tapie-Grime, M., (1995), « La place des usagers dans les choix d'infrastructures publiques ", in : Quin C. (dir.), L'Administration de l'équipement et ses usagers, Paris, La Documentation Française, pp. 255-279.

Wolton, D., (2000), « Le local, la petite madeleine de la démocratie », Hermès, n 26-27, pp. 89-97.

\section{NOTES}

1. Comité d'Évaluation et de Suivi, «Note sur la participation des habitants» («Pour une participation effective des habitants aux projets de renouvellement urbain. Proposition du Comité d'Évaluation et de Suivi de l'ANRU »), Agence Nationale pour la Rénovation Urbaine, juin 2006 (document consultable sur le site de l'agence : http://www.anru.fr/La-participationdes-habitants.html. Page consultée le 6 juillet 2008).

2. Les deux expressions de rénovation urbaine et de renouvellement urbain apparaissent dans les documents sans qu'il y ait forcément une intention précise à faire usage de l'une des deux préférentiellement, bien que la distinction puisse être en certains lieux pleinement assumée. En ce qui nous concerne, nous emploierons ici l'un et l'autre de manière indifférenciée.

3. En collaboration avec Bernard Delforce (Gériico).

4. Le matériau étudié comprend quatre dossiers et une quarantaine d'articles de magazines municipaux, plus trois brochures spécifiques. En ce qui concerne le questionnaire: parmi les 12 répondants, on compte 8 directeurs, chefs de projet ou chargés de mission sur le renouvellement urbain, 2 directeurs généraux des services, 1 chef de service administratif et une directrice de cabinet. Ce travail de première analyse a donné lieu à restitution lors de deux demijournées de rencontre professionnelle à l'IREV (une bonne trentaine de participants) en avril et juin 2007.

5. Quelques extraits choisis reflétant autant de manières de dire pour signifier que les leçons du passé ont été tirées, sans forcément faire dans le procès : « fini la jungle urbanistique »; « il s'agit de corriger les erreurs d'urbanisme des années $1970 »$; «nous allons tourner la page de ces 'grands' ensembles des années 60 ", explique ainsi un maire, « ils ont, certes, répondu à une forte demande et à la crise du logement de l'époque : des milliers de familles y ont vécu des moments heureux avant que la situation ne se dégrade, mais ils ne correspondent plus aux modes de vie d'aujourd'hui ».

6. «Ce fut l'occasion pour quelques habitants, est-il expliqué en présentation d'un article d'un magazine municipal, d'exprimer leurs craintes et leur impatience face au projet de rénovation urbaine de leur quartier dont les travaux, sur le terrain, tardent à se concrétiser. Ce fut l'occasion également pour le Maire de les rassurer sur l'évolution actuelle du dossier ANRU... et de remettre 
quelques pendules à l'heure ». Nous avons également retrouvé cet objectif de réassurance dans les réponses au questionnaire exploité dans le cadre de notre collaboration avec l'IREV.

7. Lettre d'information consultable sur le site de l'ANRU (http://www.anru.fr/-JournalRenovation-Urbaine-.html?rubrique101=\&var_mode=recalcul\&revues=375). Page consultée le 30 mai 2008.

8. Voir note 5 .

9. C'est alors plutôt la presse quotidienne régionale qui, dans les comptes-rendus de ces réunions parfois agitées, se fait l'écho de ces difficultés ressenties, la presse municipale se situant du côté de ce que nous avons appelé l'habitant projeté.

10. Ainsi que l'avancent M. Barthélémy et L. Quéré $(1995,293)$ sur les enquêtes d'utilité publique, «des responsabilités sont établies en rapport avec une certaine manière d'être affecté par les menaces que représente le projet pour le cadre de vie».

11. «Pas d'action publique sans une historicisation des événements et des situations où s'origine le quartier » ainsi que l'avance L. Quéré $(1995,117)$.

\section{RÉSUMÉS}

En explorant le discours institutionnel relatif à la concertation dans les projets de renouvellement urbain, les auteurs s'intéressent d'une part à la manière dont est mise en scène et en sens ladite concertation et s'attachent, d'autre part, à discerner les différentes figures de l'habitant portées de façon plus ou moins explicite par ce discours. Ils en viennent à montrer comment les démarches de concertation, telles qu'elles apparaissent, dans ce discours, intriquées dans des pratiques de communication, peuvent être entendues non comme moment communicationnel de production d'un neuf, pourtant ambition affichée par l'ANRU (Agence nationale de la rénovation urbaine), mais comme procédures de «mise en acceptation » d'un projet longuement élaboré en amont. L'article montre également comment l'habitant, «figuré » et « projeté » sous des statuts multiples, se voit attribué une identité pré-définie et envisagé dans un rôle circonscrit par le cadre institutionnel.

In examining the institutional discourse of dialogue about urban renewal projects in France, the authors are interested in how that dialogue is signified and presented. They distinguish several representations of residents carried by this discourse, whether explicitly or not. They show how the moves in these dialogue steps as they appear in this discourse as blending with communication practices, must be understood as acceptance setting for a project built a long time ago rather than as a communication act during which new proposals are made (even though this is a stated aim of the National Agency for Urban Renewal). The paper also explains how the resident is projected as having a multiple status and identified in a restricted number of roles and functions by this institutional framing. 
INDEX

Keywords : dialogue, inhabitant, public sphere, institutional discourse, urban renewal, territorial development

Mots-clés : concertation, habitant, espace public, discours institutionnel, renouvellement urbain, développement territorial

\section{AUTEURS}

\section{JACQUES NOYER}

Jacques Noyer est Professeur des Universités en Sciences de l'Information et de la Communication à l'Université Charles-de-Gaulle - Lille 3. Il est responsable, au sein de GERIICO, de l'équipe « Meddiatics ». Ses recherches portent, notamment, sur le rôle et la place des médias, et de leurs discours, dans la construction des problèmes publics. Il y privilégie l'approche en termes de processus de médiatisation, d'interactions au sein de l'espace public et de pratiques discursives des acteurs.

\section{BRUNO RAOUL}

Bruno Raoul est Maître de Conférences en Sciences de l'Information et de la Communication à l'Université de Lille 3 et membre de GERIICO (équipe Méddiatics). Ses domaines de recherche portent essentiellement sur les questions relatives au développement local et régional et aux mutations des territoires et sur les modalités d'insertion des technologies de l'information et de la communication dans les territoires. Il est responsable du parcours de master « Communication et développement des territoires » (UFR Infocom - Lille 3). 